\title{
RETRACTED ARTICLE: Length-weight relationship and condition factor of nine possible crosses of three stocks of giant freshwater prawn, Macrobrachium rosenbergii from different agro-ecological regions of India
}

\author{
P. L. Lalrinsanga • Bindu R. Pillai - Kanta D. Mahapatra • \\ Lopamudra Sahoo $\cdot$ Raul W. Ponzoni • Nguyen Hong Nguyen • \\ Swagatika Mohanty - Swagatika Sahu - Sovan Sahu • Vijay Kumar • \\ Gunamaya Patra $\cdot$ Shivani Patnaik
}

Received: 13 January 2012/Accepted: 11 September 2012/Published online: 22 September 2012 (C) Springer Science+Business Media Dordrecht 2012

This article has been retracted at the request of the Editor-in-Chief. The data in this article was used without acknowledgment or approval by the research team.

\footnotetext{
P. L. Lalrinsanga $(\bowtie) \cdot$ B. R. Pillai · K. D. Mahapatra · L. Sahoo · S. Mohanty · S. Sahu · S. Sahu V. Kumar · G. Patra - S. Patnaik

Central Institute of Freshwater Aquaculture, Bhubaneswar 751002, Orissa, India e-mail: viensky2@gmail.com
} 